\title{
Bending insensitivity of fiber Bragg gratings in suspended-core optical fibers
}

\author{
Charles M. Jewart, ${ }^{1}$ Tong Chen, ${ }^{1}$ Eric Lindner, ${ }^{2}$ Julia Fiebrandt, ${ }^{2}$ Manfred Rothhardt, ${ }^{2}$ \\ Kay Schuster, ${ }^{2}$ Jens Kobelke, ${ }^{2}$ Hartmut Bartelt, ${ }^{2}$ and Kevin P. Chen ${ }^{1, *}$ \\ ${ }^{1}$ Department of Electrical and Computer Engineering, University of Pittsburgh, \\ 3700 O'Hara Street, Pittsburgh, Pennsylvania 15261, USA \\ ${ }^{2}$ Institute of Photonic Technology, Department of Fiber Optics, Albert Einstein Str. 9, 07745 Jena, Germany \\ ${ }^{*}$ Corresponding author: pec9@pitt.edu
}

Received August 25, 2011; accepted September 6, 2011;

posted October 14, 2011 (Doc. ID 153456); published November 21, 2011

\begin{abstract}
This Letter presents simulation and experimental results that explore bending insensitivity of fiber Bragg gratings in suspended-core optical fibers. The implementation of thin silica bridge in the fibers enhances index contrast of the fiber core and reduces bending-induced strain transfer to the fiber core. This fiber design lead to a reduction of over 7 times in strain-induced fiber Bragg grating resonant peak shifts in the suspended-core fiber compared with that in standard telecommunication fiber, and an $0.14 \mathrm{~dB}$ bending loss at a bending radius of $6.35 \mathrm{~mm}$. (c) 2011 Optical Society of America

OCIS codes: $\quad 060.2370,060.3735,060.4005,280.4788,000.4430$.
\end{abstract}

Over the last decade, air-hole microstructured fibers have been used extensively for sensing applications. It has been shown by many researchers that proper design and fabrication of air-hole matrices in the fiber cladding or in the fiber core can lead to fiber sensors with improved sensitivity and specificity compared with those based on traditional solid-core and solid-cladding fibers. For example, proper design of air holes in the fiber cladding can lead to significant improvement in pressure sensing [1]. Fiber with a photonic crystal core can be used to separate the temperature response from the strain response of fiber Bragg gratings [2]. In light of these successes, there are ongoing efforts in the fiber optic community to explore various air-hole matrices in optical fibers to improve the susceptibility of microstructured fibers for sensing applications.

From the opposite perspective, it is also worthwhile to note that similar design approaches of air-hole matrices in a fiber's core and cladding could be applied to produce another class of fiber and fiber devices, the devices that are immune to some or all environmental fluctuations. Although totally unfit for sensing applications, these microstructured fibers and devices could find uses in optical communication, navigation, and antitampering applications. This approach could be viewed as a selfpackaging technique by fiber design. For example, an air-hole matrix can be built into the fiber structure to dampen external impacts and ultrasonics from reaching the fiber cores. This leads to environmentally robust fiber devices and systems useful for communication and navigation applications.

In this Letter, we explore this perspective by demonstrating a fiber Bragg grating (FBG) device that is over 1 order of magnitude more insensitive to fiber bending in terms of both strain transfer and optical bending loss than those in a standard telecommunication fiber (Corning SMF-28). Mechanical bending is one of the most common environmental factors that influence performance of communication and navigation devices [3] .

A four-hole suspended-core fiber (SCF) is used in this work. It is fabricated with stack and draw technology [ㅁ] as shown in Fig. 1. The diameters of the fiber and the air holes are $123 . \overline{2}$ and $25 \mu \mathrm{m}$, respectively. The $3.3 \mu \mathrm{m}$ diameter all-silica fiber core is connected with the outer shell with four thin silica bridges with a thickness of $0.9 \mu \mathrm{m}$.

This fiber was originally created for chemical sensing applications by exploring the evanescent wave propagating in the air holes [5]. The weak connections between the fiber core and the outer shells lead to optomechanical responses in the fiber core under bending that are different from those observed in solid fibers. This intuition was first explored via a numerical simulation using a finite element analysis (FEA) package ANSYS.

To accurately account for the strain distribution in the suspended fiber core through a thin silica bridge, a solid three-dimensional model was created, rather than a beam model. The model was a created using the PLANE183 element and then extruding the model with SOLID186 elements. Attention was given to ensure that the models were symmetric about the $x$ and $y$ planes with consistently even meshing to ensure that a proper solution was obtained. The model was extruded at a length of $1 \mathrm{~cm}$ with 16 elements along the length $(L)$ of the model.

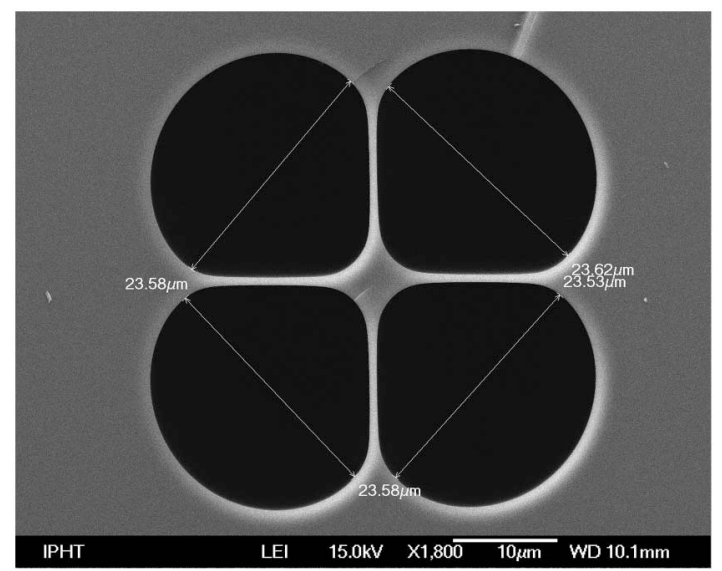

Fig. 1. Scanning electron microscopy photograph of the four-hole SCF. 


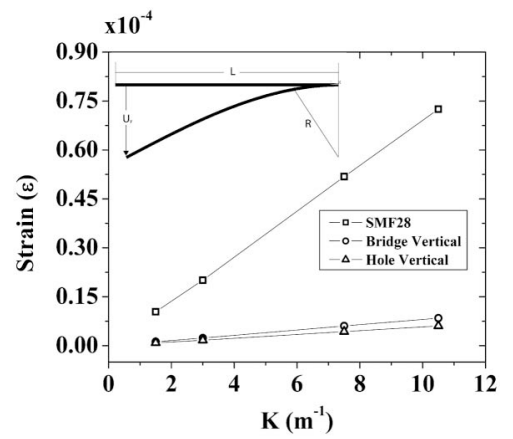

Fig. 2. FEA simulation of the bending-induced strain in SCFs. The inset shows the simulation geometry.

The fixed end of the model was constrained to allow no displacement or rotation, with the free end of the fiber having an increasing displacement $\left(U_{y}\right)$ applied, which resulted in a decreasing radius (increasing curvature). Figure 2 (inset) presents a view of the fiber model used in this paper. The results of the FEA simulation are presented in Fig. 2. What is immediately apparent is that SMF-28 has a significantly higher sensitivity to applied bending than does the SCF, with the SMF-28 having 8.59 times more induced strain. The two orientations of the SCF also have different responses to bending, with the silica bridge parallel to the bending axis experiencing a 1.4 times increase in strain over that when the air holes are parallel to the bending axis. When the outer shell of the fiber was bonded to a cantilever under bending, the large air holes allow the suspended cores to have more degrees of freedom to relax the strain.

To verify the simulated results, a bending experiment was performed on the SCF and SMF-28 with FBGs with a length of $1 \mathrm{~cm}$. Because of the pure silica material of the fiber, conventional UV writing of Bragg gratings is not possible and hydrogen loading is also difficult because of the fast out diffusion of the small core region. Therefore, all gratings were generated by using a femtosecond laser inscription technique [6]. A frequency tripled Ti:sapphire laser working at $266 \mathrm{~nm}$ together with a Talbot interferometer configuration detailed in [6] was used. The fabricated gratings had a spectral width between 0.4 and $0.6 \mathrm{~nm}$ and a reflectivity between $50 \%$ and $70 \%$. The FBGs in SMF-28 were written using a standard phase mask inscription technique with a $248 \mathrm{~nm} \mathrm{KrF}$ laser. To enhance the photosensitivity, fibers were hydrogen loaded at $1900 \mathrm{psi}$ for one week at room temperature. After the excimer laser exposure, the gratings in SMF-28 were then annealed at $120^{\circ} \mathrm{C}$ for $24 \mathrm{~h}$ before experiments.

The bending of the fibers was performed by first mounting the SCF on a rotational stage to adjust the orientation of the fiber to the bending axis. The orientation of the air holes was via a CCD microscope mounted at the end of the fiber. The fiber was then fixed via epoxy to a stainless steel cantilever $12.88 \mathrm{~cm}$ in length with the length of fiber containing the FBG placed $3.08 \mathrm{~cm}$ from the fixed end of the cantilever. This setup allowed for deflection only at the far end of the cantilever, while ensuring that the grating would be in the nonlinear bending region of the cantilever. The fiber was then bent with an increasing curvature. The FBG spectral changes were monitored by using a broadband amplified stimulated

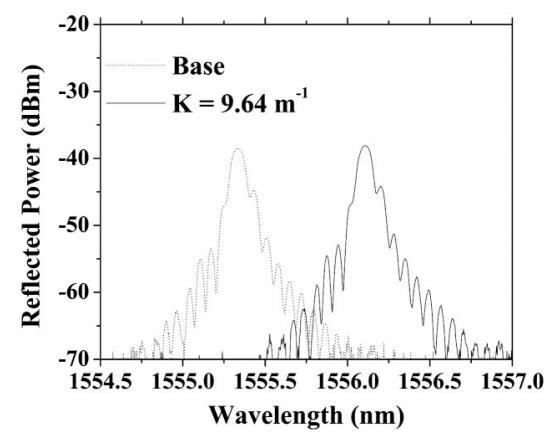

Fig. 3. Unloaded and bended FBG spectra in SMF-28.

emission source, an optical spectrum analyzer, and a circulator. This procedure was repeated for both orientations of the SCF and the SMF-28. The reflection spectra for the FBG inscribed in SMF-28 is shown in Fig. 3 as the benchmark. Bending with a radius of curvature at $9.64 \mathrm{~m}^{-1}$ led to a $0.771 \mathrm{~nm}$ FBG peak shift corresponding to a $636 \mu \varepsilon$.

The SCF with the air holes and silica bridges parallel to the bending axis for a curvature of $\sim 9.7 \mathrm{~m}^{-1}$ are presented in Fig. 4.

The SCF experienced shifts of 0.0877 and $0.0481 \mathrm{~nm}$ for the silica bridges and air holes parallel to the bending axis, respectively. There are decreases of 8.79 and 16.02 times in the wavelength shifts for the SCF having the silica bridges and air holes parallel to the bending axis, respectively. Figure 5 compares bending experimental results of FBGs in SMF-28 and in SCF with the silica bridges and air holes perpendicular to the bending axis, respectively. When the bending curvature was smaller than $5 \mathrm{~m}^{-1}$, strain-induced refractive index changes in both fibers and both orientations scaled linearly with the bending curvature. The index changes in the SCF core

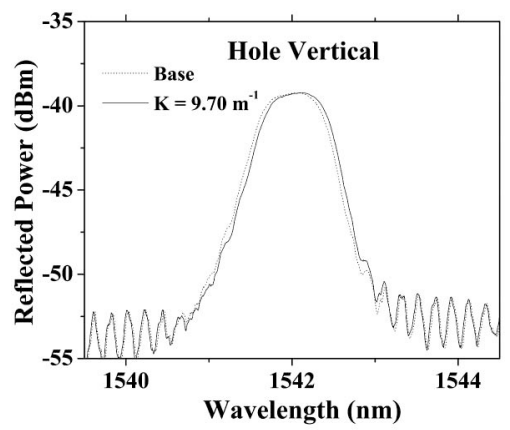

(a)

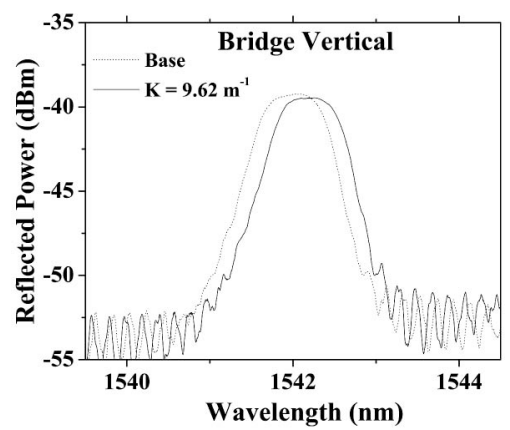

(b)

Fig. 4. Flat and bended FBG spectra in SCF with air holes and silica bridge perpendicular to the bending axis. 


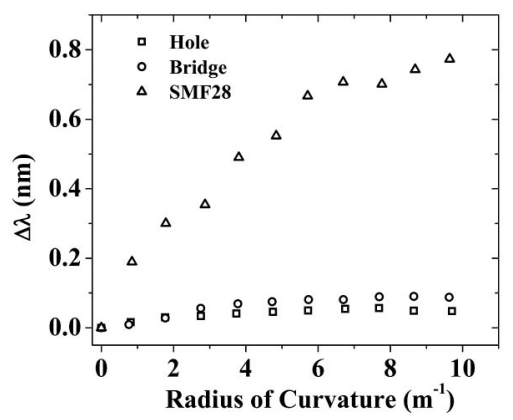

Fig. 5. Experimental results for the wavelength of the FBG shift versus bending curvature.

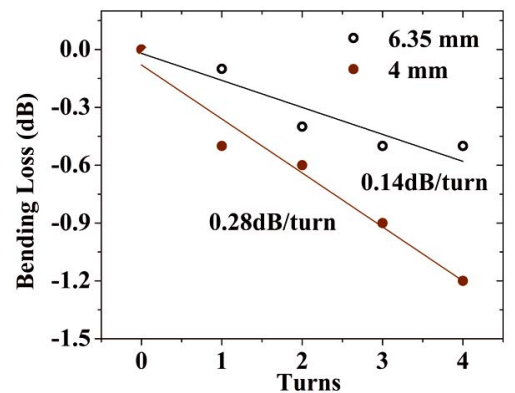

Fig. 6. (Color online) Experimental results for the bending loss of SCF versus the number of turns.

were 7.36 (bridge vertical) and 12.1 times (hole vertical) less than those in SMF-28. These are in good agreements with the simulation results. Further bending experiments beyond $5 \mathrm{~m}^{-1}$ showed a deviation from the linear trends and simulation results. The cause of this discrepancy is unclear, but is probably due to the slipping of fiber from the bending beam. However, the reduction of straininduced index changes in the SCF core from those in SMF-28 remained unchanged as 7.36 and 12.1 times for both orientations.

Another interesting trait of the SCF fiber is its low bending loss, which is important for many fiber optics devices used in navigation, communications, and fiber lasers. This is somewhat expected due to the large index contrast between the pure silica core and the air cladding. Figure $\underline{6}$ shows the optical loss measurement on SCF with bending radii of 6.35 and $4 \mathrm{~mm}$, respectively. The $0.28 \mathrm{~dB}$ /turn bending loss with a bending radius of
$4 \mathrm{~mm}$ is a significant improvement from the conventional bending-insensitive fiber, but worse than that recently reported for nanocomposited fiber [7].

In summary, this Letter explored the prospects of airhole microstructured fibers designed to be immune to applied bending. Bending loss and bending-induced strain in SCF was studied. The thin silica bridges enable high refractive index contrast fibers that mimic silica nanowires, but with much improved malleability, leading to a drastic reduction of bending loss. The same silica bridge also reduces strain transfers from the outer shell to the silica fiber core, which reduces the influence of environmental fluctuation on the fiber core. The combined advantages of low bending loss and low bendinginduced strain found in this fiber might lead to some interesting applications. Along this thread, other air-hole fiber designs might further reduce the sensitivity of fiber devices to environmental fluctuation.

This work was supported by grants from the National Science Foundation (NSF) (NSF0644681 and NSF0923006); C. M. Jewart is supported by the NSF Integrative Graduate Education and Research Traineeship Program (NSF0504345). Funding by the German state of Thuringia (Ministry of Education and Cultural Affairs) is gratefully acknowledged.

\section{References}

1. C. Jewart, Q. Wang, J. Canning, D. Grobnic, S. Mihailov, and K. P. Chen, Opt. Lett. 35, 1443 (2010).

2. C. Martelli, J. Canning, N. Groothoff, and K. Lyytikainen, Opt. Lett. 30, 1785 (2005).

3. X. Chen, C. Zhang, D. J. Webb, K. Kalli, and G. D. Peng, IEEE Photon. Technol. Lett. 22, 850 (2010).

4. K. Schuster, J. Kobelke, A. Schwuchow, M. Leich, M. Becker, M. Rothhardt, U. Röpke, J. Kirchhof, H. Bartelt, and T. Geernaert, Proc. SPIE 6588, 658804 (2007).

5. A. S. Webb, F. Poletti, D. J. Richardson, and J. K. Sahu, Opt. Eng. 46, 010503 (2007).

6. M. Becker, J. Bergmann, S. Brückner, M. Franke, E. Lindner, M. W. Rothhardt, and H. Bartelt, Opt. Express 16, 19169 (2008).

7. M. J. Li, P. Tandon, D. C. Bookbinder, S. R. Bickham, M. A. McDermott, R. B. Desorcie, D. A. Nolan, J. J. Johnson, K. A. Lewis, and J. J. Englebert, J. Lightwave Technol. 27, 376 (2009). 